\title{
DESARROLLO Y VALIDACIÓN DE UN MÉTODO ESPECTROFOTOMÉTRICO/COLORIMÉTRICO PARA LA DETERMINACIÓN DE CROMO (III) EN LICORES RESIDUALES DE PIQUELADO- CURTIDO
}

Carlos Ramiro Escalera, Luis Arteaga, Ariel Baldivieso y Rodrigo Vega

\section{RESUMEN}

Dos métodos no destructivos fueron desarrollados y validados para la determinación de concentraciones de sales de cromo en licores residuales de curtido que se producen en la fabricación de cueros: Un método espectrofotométrico y un método colorimétrico derivado. Los dos métodos se basan en el análisis de espectros de absorbancia-longitud de onda generados por soluciones patrón que fueron preparadas con los mismos reactivos químicos comerciales utilizados por una curtiembre local, en el intervalo de longitud de onda de 190-800 nm (UV-visible). Ambos métodos satisfacen los criterios fundamentales de validación: proporcionalidad (buena linealidad y buena sensibilidad) dentro del intervalo de concentraciones de sales de cromo que se observa en los licores residuales de curtido; precisión (buena repetibilidad/reproducibilidad); exactitud (sin diferencias estadísticas respecto a métodos oficiales) y selectividad (buena especificidad para la sal de cromo). Estos métodos son prácticos por su simplicidad, bajo costo y rapidez. Requieren equipos de bajo costo (un espectrofotómetro de rango visible o un colorímetro fabricado manualmente), usan los mismos reactivos químicos que se aplican en procesos de curtido corriente y no requieren de condiciones de seguridad especiales ni personal especializado. Los métodos pueden ser utilizados para el control de reciclado directo de licores de curtido-piquelado, facilitando de esta manera la adopción de esta técnica de Producción Más Limpia por parte de los fabricantes de cueros, especialmente curtiembres de escala pequeña y mediana.

Palabras Clave: Espectrofotometría, Colorimetría, Validación de Métodos, Licores de Curtido al Cromo, Cr(III). 